\title{
Peripheral Neuropathy after Open Abdominal Surgery with Self-Retaining Retractors. A Systematic Review of Randomised and Non-Randomised Clinical Trials
}

\author{
Prathima Chowdary', Monika Baumann² \\ ${ }^{1}$ Waitemata District Health Board, Auckland, New Zealand \\ ${ }^{2}$ Fisher \& Paykel Healthcare Ltd., Auckland, New Zealand \\ Email: prath1mak@yahoo.co.in
}

How to cite this paper: Chowdary, P. and Baumann, M. (2017) Peripheral Neuropathy after Open Abdominal Surgery with SelfRetaining Retractors: A Systematic Review of Randomised and Non-Randomised Clinical Trials. Open Journal of Obstetrics and Gynecology, 7, 800-814.

https://doi.org/10.4236/ojog.2017.78081

Received: May 23, 2017

Accepted: July 30, 2017

Published: August 2, 2017

Copyright $\odot 2017$ by authors and Scientific Research Publishing Inc. This work is licensed under the Creative Commons Attribution International License (CC BY 4.0).

http://creativecommons.org/licenses/by/4.0/

(c) † Open Access

\begin{abstract}
Objectives: A systematic review was undertaken to evaluate the effectiveness of incision retention available to surgeons conducting open abdominal or pelvic surgeries. Both the ability of the retractor to retain the wound and harm to the patient due to the retractor were reviewed. Methods. A search was conducted using the following databases: EMBASE, PubMed, BIOSIS, Engineering Village, Web of Science, Best practice, Science Direct, CRCnet BASE, Proquest, Wiley Online Library, and Comprehensive Biomaterial. Results. What the articles found were then narrowed down to those which matched the objective of the review. This resulted in ten articles to review. Two reviewers reviewed and summarized the articles. Femoral neuropathy was found to be a common complication due to Retractors. Other outcomes analysed or studied were found to be infected, postoperative pain and exposure provided. Femoral neuropathy can be estimated to occur at a rate between $2.6 \%$ and $7.5 \%$ in open pelvic and abdominal surgeries. The Alexis O-ring retractor was found to lower the required morphine intake following surgery when compared to the Belfour retractor. Conclusions. There is a lack of high quality/high levels of evidence studies that have been conducted on Retractors. Femoral neuropathy is the outcome most commonly documented in relation to Retractors. Surgeons need to be aware about the use and implementations of the retractors. Care should be taken in protecting the blades and during long surgery relocating retractors should be considered.
\end{abstract}

\section{Keywords}

Open, Abdominal, Pelvic, Surgery, Retraction, Nerve, Bowel, Urinary, Injury, Self-Retaining, Incision Retention 


\section{Introduction}

Iatrogenic femoral neuropathy following pelvic surgery has been known since the late 1800s. But there is no clear incidence rate or association with certain types of retractor and the modifiable factors which can decrease the incidence.

Pathogenesis is attributed to the continuous pressure exerted by the various self-retaining retractors on the pelvic sidewall and the psoas muscle.

The femoral nerve is the largest branch of the lumbar plexus arising from the dorsal divisions of the ventral rami of the second, third and fourth lumbar nerves. It comes down through the psoas muscle approximately $4 \mathrm{~cm}$ above the inguinal ligament. It at all times remains in the operative field of the surgeon who operates in the abdomen and pelvis. There is a relatively poor blood supply to the femoral nerve as it crosses the pelvis and is therefore susceptible to ischemia.

The femoral nerve $1-4 \mathrm{~cm}$ distal to the inguinal ligament separates into anterior and posterior sections. The anterior division of the femoral nerve gives off anterior cutaneous and muscular branches. The anterior division supplies the Sartorius muscle and the 2 sensory branches the intermediate and medial cutaneous nerves of the thigh. The posterior division supplies the quadriceps femoris and articularis genus. The femoral nerve also innervates the hip and knee joints.

We wanted to execute a systematic review to count at the incidence of femoral neuropathy secondary to self-retaining Retractors and the factors tied in with it. This in return should help us in the design phase of the Retractors to decrease the incidence of this debilitating iatrogenic injury (Table 1).

\section{Methods}

\section{Operational Definition of Population and Intervention:}

The population included in the review were only those who were undergoing open or laparotomy pelvic and abdominal surgeries either using or in some (control group) cases not using retractors. Though mostly adults were taken into consideration, but in few cases experience and complaints of people from all age groups have been considered. Femoral neuropathy and other problems caused by the nerves were the areas of main focus, though some comments and review has also been performed involving the universal purpose of the Retractors and the benefits and ease of them. Obese patients have also been taken into consideration and the subsequent results due to the use of Retractors have been reviewed as well.

Table 1. Signs and symptoms of Femoral nerve neuropathy.

\begin{tabular}{cc}
\hline SIGNS & SYMPTOMS \\
\hline $\begin{array}{c}\text { Inability to raise the led straight off the bed } \\
\begin{array}{c}\text { Decreased and absent sensations of touch and pain } \\
\text { over the anteriomedial aspect of the thigh and leg }\end{array}\end{array}$ & $\begin{array}{c}\text { Complaints of numbness of the } \\
\text { anteriomedial aspect of the thigh and leg }\end{array}$ \\
Decreased or absent patellar reflex & Pain localised to the anterior hip joint area \\
\hline
\end{tabular}


Mostly no intervention was thought about at the time when the procedure was performed, but post-operative interventions were noted in a few cases where special handling has been needed for the patients during the notice point.

\section{Search Strategy:}

We carried on a systematic inspection of articles found from Jan 1970 from Jan 2015. The databases used in the search were EMBASE, PubMed, BIOSIS, Engineering Village, Web of Science, Best practice, Science Direct, CRCnetBASE, Proquest, Wiley Online Library, and Comprehensive Biomaterial. The database searches were performed using the mentioned keywords. Variations on the words i.e. different endings, plural etc were used along with the appropriate logic operators (OR, AND, etc.) as required for individual databases.

\section{Inclusion and Exclusion Criteria:}

The papers were required to be peer reviewed, with English language and published between January 1970 and January 2015. The articles were to be focused on adults and must include data focused on retractor used in pelvic or abdominal surgeries. They were discussing the effectiveness of the retention provided or any injury that occurred to the patient due to the retractor. Laparoscopies and endoscopic surgeries were excluded from the review.

\section{Organization of Evidence:}

A quantitative study, critical review form was used by all reviewers when summarizing the important information on each article. This form has been developed by McMaster University, Canada [1]. The review form includes summaries of the study's purpose, whether a literature review had been completed, the design of the study and any biases that could have been operating, details of the sample sizes and characteristics and ethics approvals. The measure and outcomes, interventions, results, including statistical significance, patient dropouts and clinical importance, conclusions and implications were featured in the form. The reviewers used this form to review the articles chosen to be included in the study. The articles were equally distributed among the reviewers. Where reviewers disagreed in the form, the article was reviewed by one of the other two reviewers. Along with the form, the articles were ranked according to "Sackett's levels of evidence and grades of recommendation" as explained by American Academy for Cerebral Palsy and Developmental Medicine (AACPDM) [2].

Typically, reviews only included studies with levels of evidence between I and $\mathrm{V}$. In this review, all levels of evidence were included due to the nature of what was researched. Many of the retractors in use have been used since the 1970s. Our searches have shown that randomized controlled trials or other higher level of evidence study types has been rare for Retractors. This is likely to be due to a lack of motivation or need when the retractors were first developed and over the long period for which they have been in use, the detractors will be seen as a useful and necessary tool, but one that would not benefit from expensive or indepth studies. Most articles related to retractors have been retrospective analyses and have therefore been included. 


\section{Results}

The results of the searches are given in Figure 1-participant flow diagram.

Two papers were excluded from the study once the articles were read in full because, despite their abstracts, they were found to not match the review's requirements. One article explained how to use a retractor with strong bias towards the retractor [3]. No survey or trial was acquitted and there was no clinical support of the retractor. There was therefore no evidence for the retractor's effectiveness in retaining the wound or any patient effects.

The second paper was excluded as it discussed a novel surgical procedure of a "mini laparotomy" [4]. The only mention of retractors was related to their placement and replacement during the surgery, but no data, evidence or comment were made on the effectiveness of retention or any effect on the patient due to the retractor use.

This resulted in a total number of articles to be reviewed be ten. A summary of the articles can be found in Table 2 .

\section{Study Characteristics.}

A total of at least 12,880 surgeries were used in the ten articles considered in this review. Three of these studies were random control trials [5] [6] [7], two were retrospective and prospective analyses [8] [9] with the remaining five being retrospective analyses. Of these cases, two were chosen not to be included in the statistical summaries. One paper [10] only had two cases considered and the

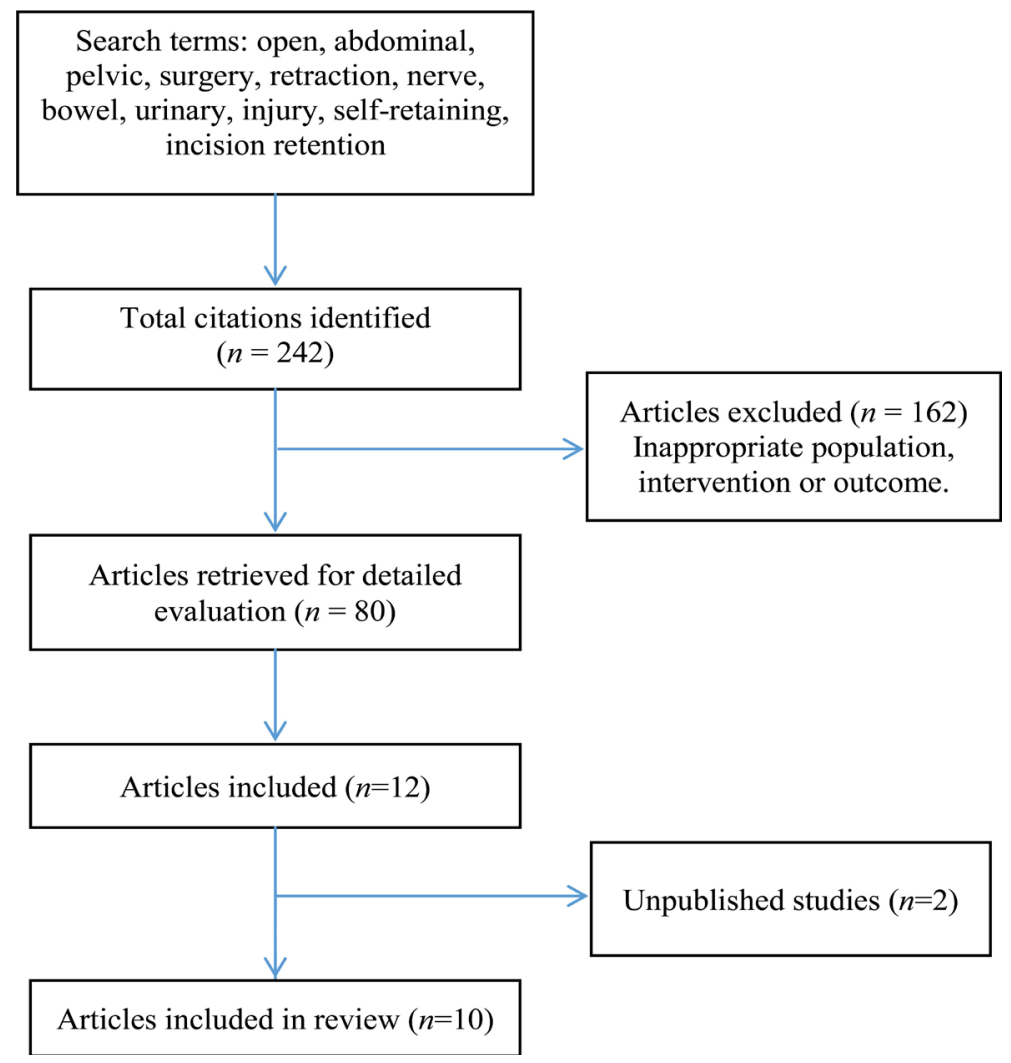

Figure 1. Flow diagram. 


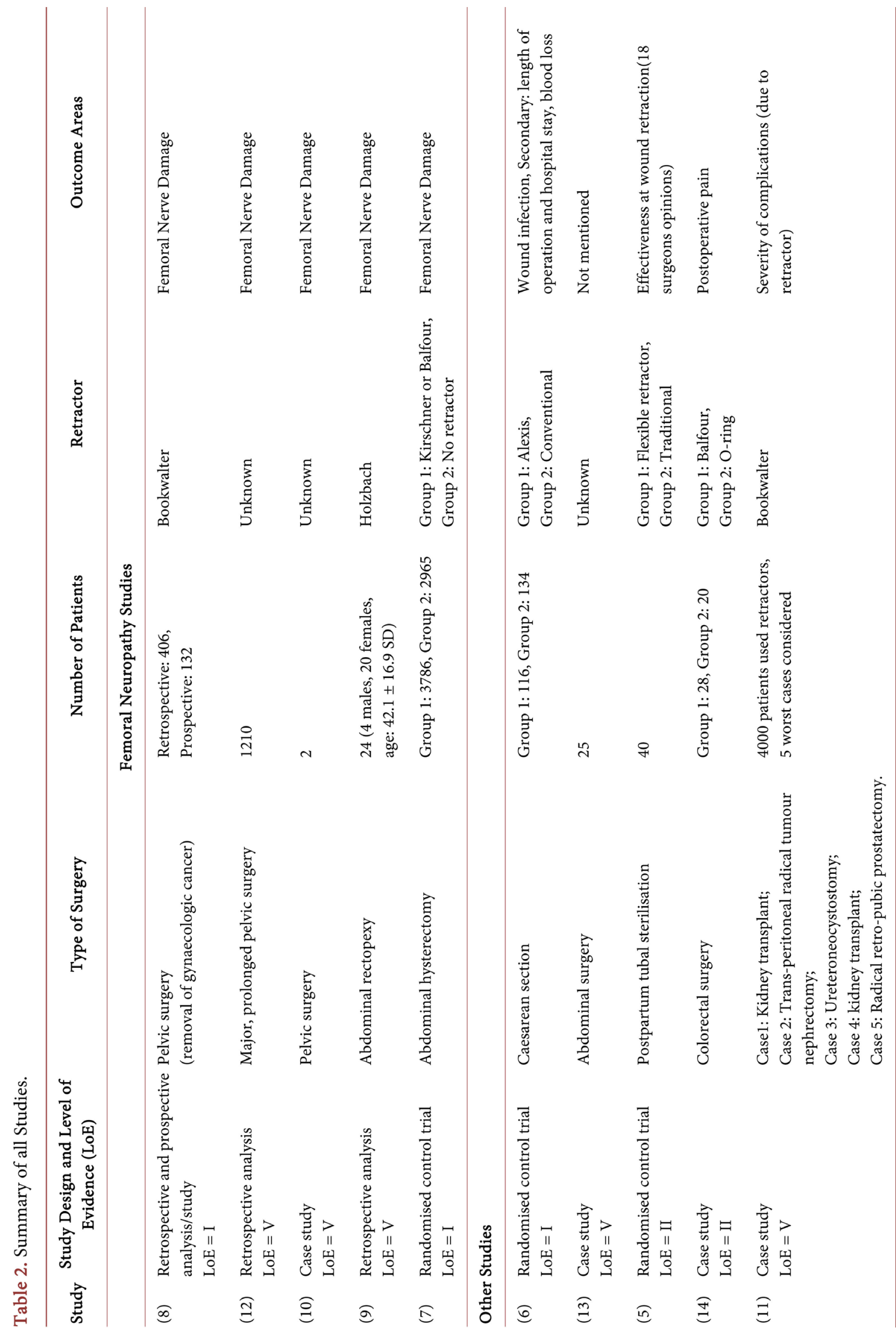


other [11] reviewed 4000 surgeries and discussed the five cases with the worst patient outcomes.

Five articles looked at the femoral nerve damage that was most likely caused by the retractors used during surgery [7] [8] [9] [10] [12]. Two surgeries looked at retractor used specifically with obese patients [6]-[13]. The remaining articles looked at other outcomes such as retention preference between a new and traditional retractor for a particular surgery type and cases where there were serious complications for patients due to retractors following the surgeries [5] [11] [14].

\section{Patients.}

The sample sizes for the randomised control trials (RCT) ranged from 40 patients (experimental and control combined) [5] to 6751 patients (3786 experimental and 2965 control patients) [7]. It should be noted the 40 patient RCT was a pilot study.

The other remaining studies (retrospective and both retrospective and prospective analyses) sample sizes ranged from two [10] to 4000 patients [11] reviewed. It should be noted that larger sample sizes quoted are the total number of surgeries reviewed and only a proportion of the surgeries resulted in harm to the patient due to the retractors used.

Types of Surgery:

The article selection required the surgeries to be confined to either abdominal or pelvic surgeries. Within this requirement, a wide range of surgeries have been encompassed. These include tumour resection [8] [11], caesarean section [5] [6], hysterectomy [7], abdominal rectopexy [9] and general pelvic surgeries [10] [12] [13] [14].

\section{Retractors:}

A range of retractors was used across the ten articles. They included Belfour, Bookwalter, Holzbach, Alexis O-ring, Kirschner, and a flexible retractor. In a paper [5] the brand or type of the retractor was not even specified. In some cases, where a control group was used, the control group's retractor was only specified to be a "conventional" or "traditional" retractor [5] [6] (Table 2).

\section{Outcome Measures:}

In the five articles which looked at femoral neuropathies, the main outcome measure was whether the patient experienced femoral neuropathy following surgery [7] [8] [9] [10] [12]. In some cases, data were also collected as to whether the neuropathy subsided or continued to be an issue even years later [9].

The other studies had varying outcome measures. Some of these included length of hospital stay, quantity of pain medication required postoperatively, length of surgical incisions and surgeons' surveys completed immediately after completing a surgery [5] [6] [11] [13] [14].

Data Analysis.

Table 4 includes all the data analysis included in the articles. Four of the articles commented on the statistical significance of the results for their respective studies or analyses with two having the actual "p" values [6] [7] [8] [14]. The 
three other studies reported the results without having completed a statistical analysis [5] [9] [12].

\section{Discussion}

Tables 2-4 summarize the articles reviewed. Table 2 includes all ten articles which were reviewed. Table 3 provides a matrix of results for questions used to evaluate the quality of the studies conducted. Table 4 summarizes the articles with clinically relevant results and some statistical results (as mentioned earlier). Due to the limited number of articles with good statistical analyses and RCTs, the results and discussion have been combined for fluidity. Because of the heterogenicity of the articles a meta-analysis could not be performed.

\section{Femoral Neuropathies.}

In the database search five articles were found which looked at femoral neuropathy and self-retaining retractors. One was a randomized controlled trial [7], one was a retrospective study [12] and two articles had both retrospective and prospective data in them [8] [9]. One was a case study of two cases and has been excluded from this discussion [10].

In the randomized trial the incidence was $7.5 \%$ in the group where Retractors were used [7] in which the results were statistically significant. In the retrospective group the incidence was $1.9 \%$, however, this result was not statistically significant.

In the two papers in which both retrospective and prospective data were collected, the incidence was $2.9 \%$ (statistically significant) [8] and 25\% (statistically not significant) [9].

A rough estimate of incidence can be made by looking at the articles with larger sample sizes and statistical significance can be concluded to lie between $2.9 \%-7.5 \%$. The incidence, however, is very dependent on multiple factors which include the surgery (or the complexity of it), surgeon experience (including surgeon experience with the newer self-retaining retractors), patient BMI, pelvic side wall dissection, type of anesthetic, muscle relaxation and patients reporting symptoms.

The most common cause of injury in all the articles was compression injury rather than complete transaction, nerve caught in suture material or diathermy injury.

We conclude that with Retractors that are very commonly used in pelvic/abdominal surgery, care must be taken to use smaller blades when possible, to only retract muscle and to place a wet swab behind the blades.

Obese Patients.

Two papers were found which included retractor used for obese patients. One article was a randomised control trial evaluating surgical infection after C-section using an Alexis O-ring retractor (AOCSR) and a control group utilising a conventional self-retaining retractor [6]. Although statistically significant difference was not found in infection rates between the two retractors ( $\mathrm{p}$-value $=0.76$ 


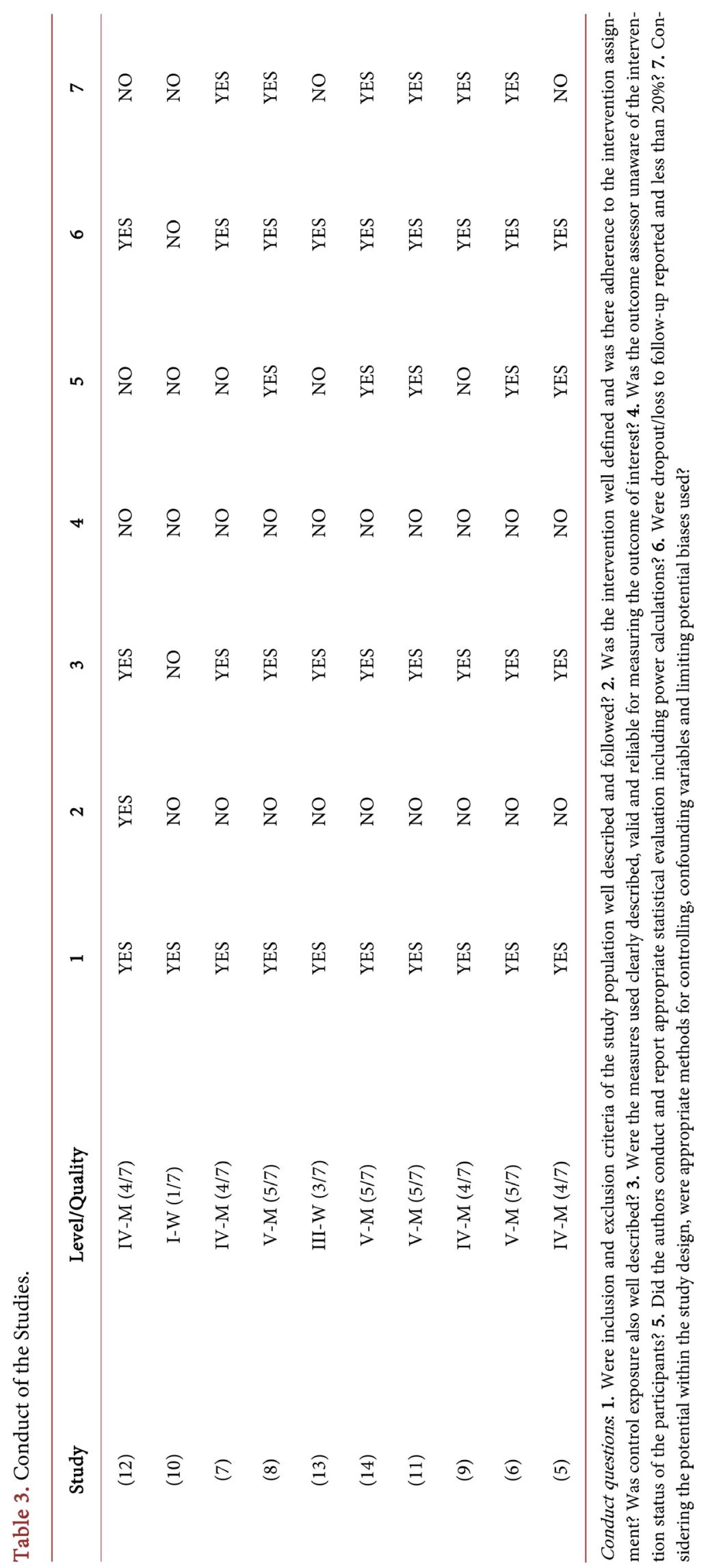




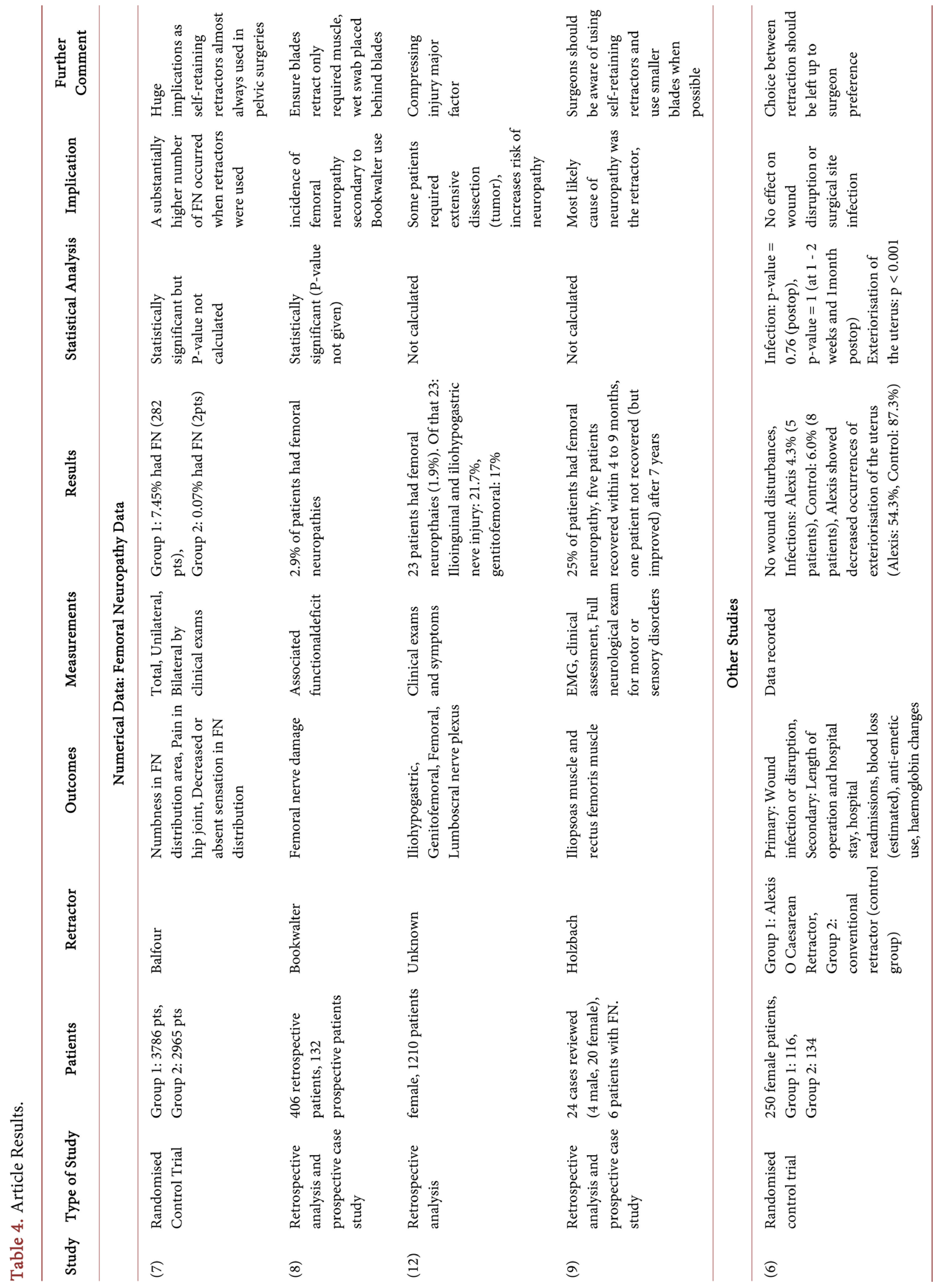



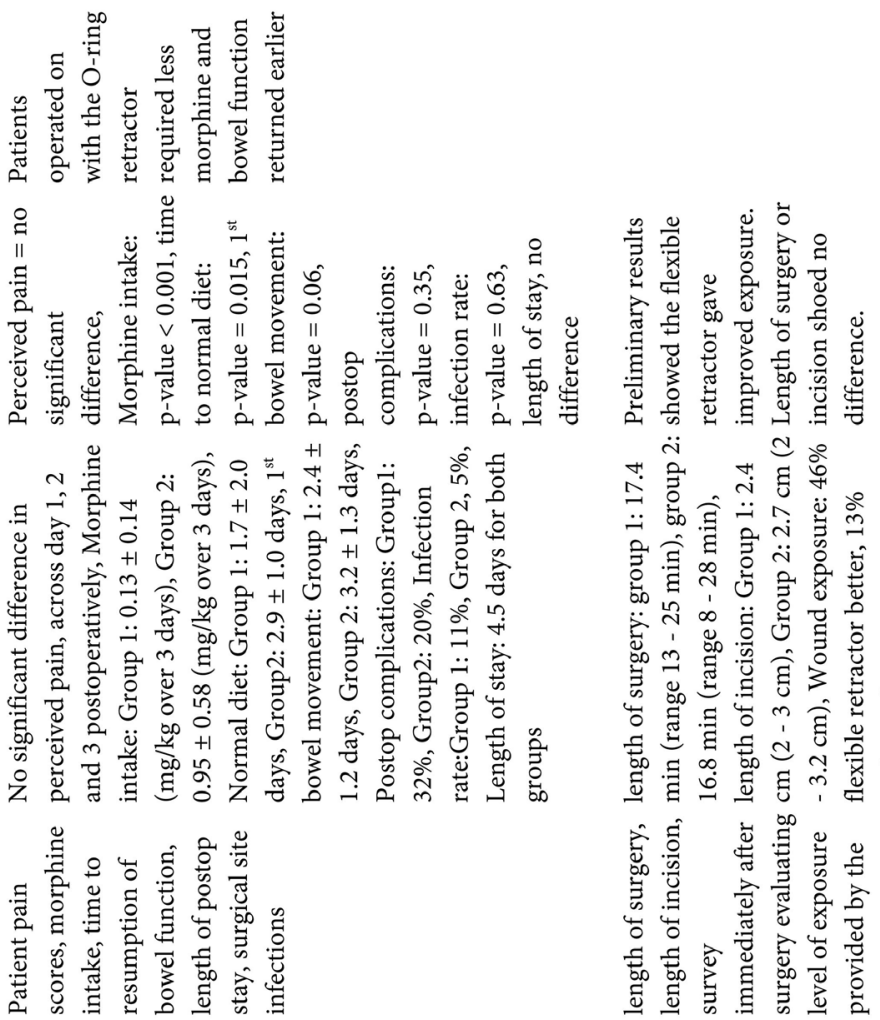

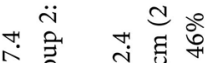

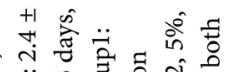

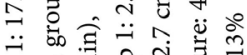

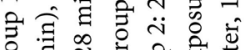
ठั.

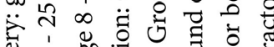

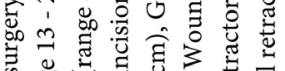
क

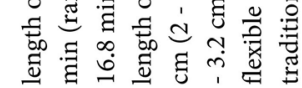

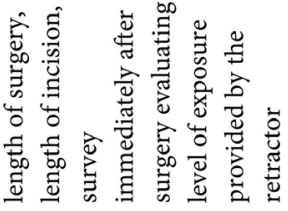

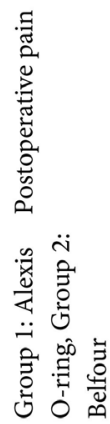

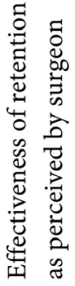

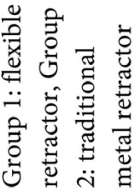

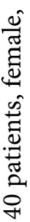
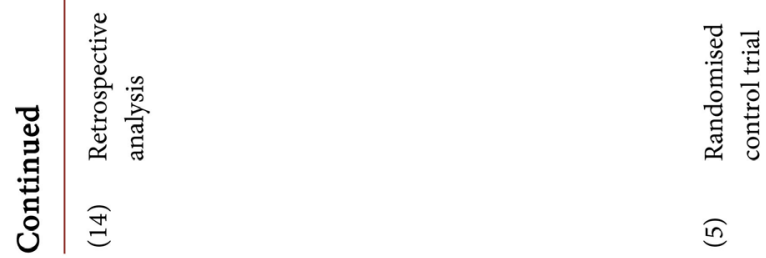

$\sqrt{6}$ 
postoperatively), there was a significant difference in the occurrences of exteriorization of the uterus (Alexis: $54.3 \%$, Conventional $87.3 \%$ ), with a p-value of $<0.001$.

The second article [13] was a series of case studies which looked at creating additional incisions to help with retractor placement in obese patients. The quality of the study was low but shows that there are or have been issues with using common retractors in obese patients.

There was little found in the search in relation to obese patient surgeries but does highlight that this may be an area that requires more studies, or this may be due to the catastrophic nature of conducting open surgeries on obese patients.

\section{Retractor Comparisons.}

Two papers were also found which included comparative studies of two different retractors and the outcome of which guided us towards an indication of the benefits of a retractor in each case. One of the papers by Lohsiriwat et al. [14] compared the Alexis O-ring retractor with that of the traditional Balfour retractor while in the second paper by Treece et al. [5] drew a comparison between a flexible self-retaining retractor and a traditional metal retractor. Postoperative pain [14] and surgery difficulty [5] were the measuring criteria of the studies.

One of the retrospective analyses [14] showed a statistically significant lower morphine intake (per kg) in patients operated on with the Alexis O-ring retractor than the Belfour with a p-value $<0.001$. Return to a normal diet and proper bowel function was seen earlier with O-ring patients than the Belfour patients with $p$ values of 0.015 and 0.06 respectively. Other outcomes such as complications and infection rates were not statistically significant.

In the randomised control trial [5] a flexible self-retaining retractor and traditional retractor were compared for their wound exposure as evaluated by surgeons. There was no statistically significant difference between the two retractors in terms of the length of surgery or length of incisions. There was a significant difference between the perceived exposures provided by the retractors. $46 \%$ of surgeons found exposure to be better with the flexible retractor while $13 \%$ believed that the traditional retractor gave better exposure.

These articles show that there is potential benefit to the use of modern retractors either in patient wellbeing or of the surgeons using the devices. In both the cases the research was done over a relatively short period of time. Larger RCTs and more patients would help to gain a better indication of the level of benefit O-ring and flexible retractors have over traditional retractors.

\section{Retractor Complications.}

One article was a retrospective case series which looked at patients operated on with the Bookwalter retractor and reported on the five worst patient outcomes which occurred due to retractor use [11]. While this article cannot be reported on in terms of statistical significance, it does highlight the importance of correct use of retractors and how much of an impact this may have on patient outcomes.

One case of femoral neuropathy occurred and three cases of a perforated ce- 
cum causing peritonitis and/or distended abdomens (and in addition of one case, a retroperitoneal abscess). All required a second surgery to rectify. One of the three patients died 75 days after the initial surgery. The other two patients were discharged after 46 to 51 days after the initial surgery. The five cases were found to have fatal peritonitis, were operated on and the patients were discharged on postoperative day 54 [11]. The symptoms of the damage were not seen until 2 to 7 days after the initial surgery. This shows that while these types of complications may be rare, incorrect retractors or improper use, can cause serious patient harm. This highlights the importance of retractors and that they are not a trivial piece of equipment in the operating theatre [11].

Limitations of This Review.

Many retractors in use today have been used for possibly the past 30 to 40 years and as such have not generally been seen as medical devices worth spending time and money on significant studies. This was seen in the quality and levels of evidence of the articles found and reviewed in this systematic review. A blinded study is not a possibility with retractors. The older, commonly used retractors have not had RCTs conducted and at most had only the retrospective analyses of the surgeries. This (historically) shows the lack of interest in retractors. With newer retractors such as the Alexis O-ring retractors and flexible retractors, high quality studies are starting to be conducted. This is likely to be due to the need to show clear benefits over traditional retractors for surgeons and hospitals to be willing to change. The increase in options of retractors is another possible driver for the higher quality studies.

Additionally, the outcomes are very dependent on surgical technique, type of operation, BMI, anaesthetic and therefore difficult to apply to a general population. The operations were also vastly different, some were gynaecology surgeries while others were related to colorectal surgery. Again the wide range of surgeries were needed to be considered for this review as only one or two articles may exist for a retractor in a certain type of surgery.

This systematic review was therefore limited by the lack of studies on many common place retractors, more significantly by the lack of high quality and high levels of evidence studies that historically might not have been of much value to be conducted.

\section{Conclusions}

Femoral nerve is very susceptible to injury and ischemia because of its anatomical location in the abdomen and pelvis and its relatively poor blood supply. Wound infection is also a well-recognized complication for surgery as well as patient pain postoperatively. Effective wound exposure is obviously a key requirement of retractors, but only one article was found to analyse this.

The outcome of the database search was unable to find any type of retractors that were superior to others in preventing/reducing femoral nerve damage or wound infection rate. Retractors that were found to have benefits over conven- 
tional/traditional retractors were only found to be beneficial in one aspect, with other outcomes not been assessed or found in other articles.

The choice of retractors should be dependent on the experience of the surgeon with the retractor that they are using, surgery and BMI of the patient.

More randomised controlled trials or good quality prospective studies are required to look at the various retractors available to surgeons to help determine the risk of nerve injury and infection.

One of the main points to be noted is that recognition and immediate management of a complication is of upmost importance. In all the studies looked at, patients who had their injury recognised early and a multidisciplinary approach was taken recovered much better from the neuropathy than those with a delayed diagnosis. Only two studies were statistically significant and could be included to calculate incidence.

With the emergence of new self-retaining retractors such as Alexis $\mathrm{O}$ retractor, it is important to understand that even though it does make exposure better in obese patients, the one RCT analyzed, which was statistically significant did not show a decrease in infection rate among the Alexis retractor group.

It is important to note that risk lies on an individual basis and it is important to perform an adequate pre-operation work up of all patients and able to predict which patients are at higher risk of femoral nerve damage and consent patients accordingly. In the studies that we looked at, the higher BMI patients were at higher risk. It was noted that it happened when the blades were too deep and were retracting the psoas muscle. Also use of adequate muscle relaxation by the anaesthetist played a major role in preventing nerve injury. Correct placement of swabs and releasing the tension on the retractors when not operating in that field were also important factors that were recognised in the studies.

For surgeons who perform abdominal and pelvic operations, it is important to take note that there is a risk of femoral neuropathy and to take steps to try to minimize this. It is also important to perform the required tests and investigations necessary if there is a suspicion of injury and to treat appropriately.

\section{Disclosures}

Prathima Chowdary and Monika Baumann have no conflict of interest related to this study.

\section{References}

[1] Law, M.S. (n.d.) Occupational Therapy Evidence-Based Practice Therapy-Quantitative Review Form.

http://www.srs-mcmaster.ca/ResearchResourcesnbsp/ResearchGroups/CentreforEvi denceBasedRehabilitation/EvidenceBasedPracticeResearchGroup/tabid/630/Default. $\underline{\text { aspx }}$

[2] Darrah, J.H. (2008) AACPDM Methodology to Develop Systematic Review of Treatment Interventions (Revision 1.2).

[3] Glaysher, M.M. (2014) Reversal of Ileostomy Utilising Lone Star Retractor System. 
Techniques in Coloproctology, 18, 1125-1126. https://doi.org/10.1007/s10151-014-1202-4

[4] Benedetti-Panici, P.M. (1996) Surgery by Minilaparotomy in Benign Gynecologic Disease. Obstetrics \& Gynecology, 87, 456-459.

[5] Treece, Y.Z. (2006) Randomized Trial to Evaluate the Use of a Flexible Self-Retaining Retractor for Postpartum Tubal Sterilization: A Pilot Study. Contraception, 74, 181. https://doi.org/10.1016/j.contraception.2006.05.013

[6] Childress, K.S. (2015) The Effect of a Barrier Self-Retaining Retractor in Obese Patients Undergoing Cesarean Section. American Journal of Obstetrics \& Gynecology, 212, S248.

[7] Goldman, J.A. (1985) Femoral Neuropathy Subsequent to Abdominal Hysterectomy, a Comparative Study. European Journal of Obstetrics \& Gynecology \& Reproductive Biology, 20, 385-392. https://doi.org/10.1016/0028-2243(85)90062-0

[8] Maneschi, F.N. (2014) Femoral Nerve Injury Complicating Surgery for Gynecologic Cancer. International Journal of Gynecological Cancer, 24, 1112-1117. https://doi.org/10.1097/IGC.0000000000000141

[9] Infantino, A.F. (1994) Femoral Nerve Damage after Abdominal Rectopexy. International Journal of Colorectal Disease, 9, 32-34. https://doi.org/10.1007/BF00304297

[10] Corbu, C.C. (2002) Femoral Nerve Palsy Caused by a self-Retaining Polyretractor during Major Pelvic Surgery. Urologia Internationalis, 68, 66-68.

https://doi.org/10.1159/000048421

[11] Noldus, J.G. (2002) Major Postoperative Complications Secondary to Use of the Bookwalter Self-Retaining Retractor. The Journal of Urology, 60, 964-967. https://doi.org/10.1016/S0090-4295(02)01946-5

[12] Cardosi, R.C. (2002) Postoperative Neuropathies after Major Pelvic Surgery. American College of Obstetricians and Gynaecology, 100, 240-244.

[13] Tera, H. (1983) Use of Abdominal Wall Retractors at Laparotomy in Grossly Obese Patients. World Journal of Surgery, 7, 555-556. https://doi.org/10.1007/BF01655951

[14] Lohsiriwat, V.L. (2014) Atraumatic O-Ring Wound Retractor Reduces Postoperative Pain. Techniques in Coloproctology, 18, 1177-1178. https://doi.org/10.1007/s10151-014-1225-x 


\section{Appendix}

Conduct questions:

1) Were inclusion and exclusion criteria of the study population well described and followed?

2) Was the intervention well defined and was there adherence to the intervention assignment? Was control exposure also well described?

3) Were the measures used clearly described, valid and reliable for measuring the outcome of interest?

4) Was the outcome assessor unaware of the intervention status of the participants?

5) Did the authors conduct and report appropriate statistical evaluation including power calculations?

6) Were dropout/loss to follow-up reported and less than $20 \%$ ?

7) Considering the potential within the study design, were appropriate methods for controlling, confounding variables and limiting potential biases used?

Submit or recommend next manuscript to SCIRP and we will provide best service for you:

Accepting pre-submission inquiries through Email, Facebook, LinkedIn, Twitter, etc. A wide selection of journals (inclusive of 9 subjects, more than 200 journals)

Providing 24-hour high-quality service

User-friendly online submission system

Fair and swift peer-review system

Efficient typesetting and proofreading procedure

Display of the result of downloads and visits, as well as the number of cited articles

Maximum dissemination of your research work

Submit your manuscript at: http://papersubmission.scirp.org/

Or contact ojog@scirp.org 